УДК 614.2

DOI 10.17802/2306-1278-2021-10-4-106-111

\title{
REMOTE CLINICAL QUALITY MANAGEMENT OF ENDOVASCULAR CARE
}

\section{A.Yu. Abramov ${ }^{1}$, D.I. Kicha ${ }^{1}$, R.S. Goloshchapov-Aksenov ${ }^{1}$, O.V. Rukodayniy ${ }^{1}$, A.M. Nazarov ${ }^{2}$,} A.G. Koledinsky ${ }^{1}$

Peoples Friendship University of Russia (RUDN University), 6, Miklukho-Maklaya St., Moscow, Russian Federation, 117198; '2 Orenburg State Medical University, 6, Sovetskaya St., Orenburg, Russian Federation, 460000

\section{Highlights}

- To gain a high level of knowledge in healthcare requires hours and hours of practice with an experienced mentor. Remote clinical management is relevant in conditions of personnel shortage or during certain periods such as pandemic of infectious diseases. Scientific novelty and practical significance of the study is the discovery of the clinical management effectiveness of remote X-ray endovascular care quality control and scientific and methodological justification of the mentor's strategic role.

To assess the effectiveness of remote clinical quality management of endovascular
care.
The system of clinical quality management of medical care in myocardial
infarction (MI) including the quality of remote control of endovascular care was
developed and introduced into the health care system of the Moscow Region as
a part of the comprehensive study in $2008-2020$. The number of people under
the study was 8375 . The ground for assessing the effectiveness of remote clinical
management in $2019-2020$ was the health care system of megapolis. Based on
the analysis of 2966 endovascular procedures protocols, the treatment tactics
effectiveness of intraoperative decisions was studied after an emergency coronary
angiography (ECA) had been performed by interventional cardiologists. The
system of remote clinical quality management of endovascular care included a
complex of audiovisual communications, computer system processes, mentoring
and the algorithm for making an intraoperative decision. The effectiveness of
remote clinical quality management of endovascular care was investigated on
the number of percutaneous coronary interventions (PCI) in MI, mortality of
patients with MI in the Regional vascular center in $2019-2020$. The T-criteria
was used to assess the reliability. The material statistical processing was carried
out in the Statistica 6.0 package calculating adequate statistical indicators and
their reliability at $\mathrm{p} \leq 0.005$.

Received: 09.07.2021; received in revised form: 16.08.2021; accepted: 14.09.2021

\section{List of abbreviations}

ECA - emergency coronary angiography PCI - percutaneous coronary interventions

\section{Introduction}

The effective health care development is associated with the integration of various clinical and management processes which are aimed at the medical care quality improvement. The management of such processes is defined as clinical management in the scientific literature.

Corresponding author: Roman S. Goloshchapov-Aksenov, mzmo-endovascular@mail.ru; address: 6, Miklukho-Maklaya St., 
The quality of remote medical care management is of particular importance as it is connected with the use of information telemedicine technologies.

The head of the regional vascular center forms and regulates the clinical and organizational processes for improving the quality of medical care in acute cardiovascular diseases.

Medical education and clinical management systems in most countries around the world have been forced to switch to a remote or mixed work format since March 2020 owing to the increase of COVID-19 cases. The growth of cardiovascular diseases, coronavirus infected patients, a shortage of qualified cardiologists and interventional radiologists advance the need of remote medical technologies to provide high-tech endovascular care.

The current prevalence of coronary heart disease in the world is 1,655 cases per 100,000 of population and it is to exceed up to 1,845 by 2030 . Eastern European countries and Russia occupy the leading position [1,2]. Acute coronary syndrome is the main cause of death of people over 65 years old. It is predicted that by 2022 the number of hospitalizations for acute coronary syndrome worldwide will increase to 1.43 million people [3].

Epidemiologists forecast that the cases of acute coronary syndrome will grow from 25.45 million in 2015 to 30.62 million in 2025 at an annual growth rate of $2.03 \%$. Such countries as the USA, France, Germany, Italy, Spain, UK and Japan are under a special threat.

In the USA ACS will be higher in men (61.81\%) over 65 years $(62.84 \%)$. STEMI, NSTEMI, and UA will constitute approximately $25 \%, 40 \%$, and $35 \%$ of acute coronary syndrome cases. In $33 \%$ of patients with acute myocardial infarction with ST segment elevation are hospitalized. $44 \%$ of patients without ST segment elevation, and $23 \%$ of patients with unstable angina pectoris are hospitalized [4].

Endovascular care is most effective in the treatment of patients with acute coronary syndrome. Nowadays the mortality rate from myocardial infarction does not exceed $8-9 \%$ in interventional cardiology departments. However, endovascular care organization on the regional level including a shortage of qualified interventional cardiologists, an insufficient number of catheterization medical centers, and public health pressures due to COVID-19 pandemic may cause a decrease in the of medical care effectiveness in acute coronary syndrome $[5,6]$. Adopting the correct strategy of endovascular care in the treatment of patients with acute myocardial infarction with ST segment elevation, of whom, according to statistics, about $33 \%$ are hospitalized, in most cases does not cause difficulties. Acute myocardial infarction with ST segment elevation in most cases is associated with occlusion or critical stenosis of the left main coronary artery or its branch from $99 \%$ of patients. This is clearly visualized during coronary angiography. An angiogram of the left coronary artery of the patient with acute myocardial infarction and with ST segment elevation caused by critical stenosis of the left anterior descending coronary artery is presented in figure 1 .

Interventional cardiologists evaluate the coronary angiography with pathological changes and electrocardiogram data and adopt the correct strategy for endovascular myocardial revascularization.

It may be difficult for the cardiology team to choose the correct treatment strategy after diagnosing a symptom-associated coronary artery in patients with acute coronary syndrome without ST segment elevation, with multiple, multilevel coronary artery disease. Clinical management of remote telehealth technologies increase the efficiency and quality of the endovascular care. It is very important to timely identify an acute coronary syndrome-associated coronary artery during coronary angiography analysis in patients with acute myocardial infarction or unstable angina, with multiple, multilevel coronary arteries lesions. Remote or face-to-face assistance by an interventional radiologist whether to perform emergency percutaneous coronary angioplasty is a strategic task in the provision of endovascular care. The modern results of studies indicate that failure to perform emergency myocardial revascularization in patients at high risk according to the GRACE scale increases the likelihood of developing cardiovascular endpoints (acute myocardial infarct or death). The results obtained in the course of this study show the effectiveness of the use of telemedicine technologies in the clinical management of endovascular care [7].

Remote health care management mentoring is an essential process of informal transfer of knowledge and psychological support. Application of remote technologies to the clinical management of endovascular care is of extremely high importance and involves

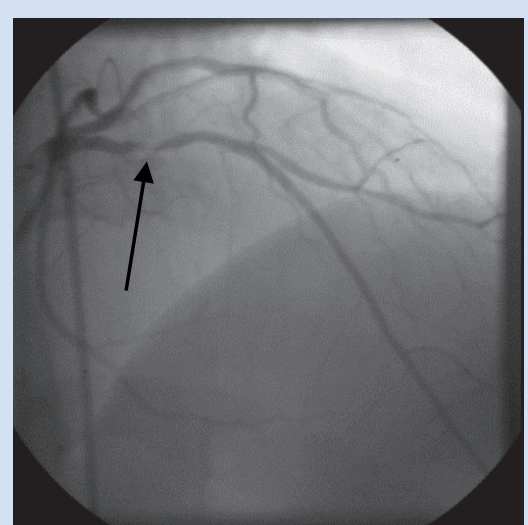

Figure 1. Coronary angiogram of the patient's left coronary artery with acute myocardial infarction with ST segment elevation. The arrow indicates a critical stenosis of the left anterior descending coronary artery 
long-term communication between the mentor and medicine team.

The mentor is the leader of the medical organization, often the head of clinical department (for example the head of cardiovascular surgery department) who supports the effective development of the process in face-to-face and remote communication mode, de-escalation of the processes of decreasing the quality of medical care and increasing the mortality rate, and protecting the interests of patients and their families $[8,9]$.

Remote quality management of medical care preserves professional resources, especially under conditions of an increased burden on health care and personnel shortages, and directs a systematic management of processes [10].

\section{Methods}

As part of a comprehensive study in 2008-2020 a system of clinical management of the quality of endovascular care in acute myocardial infarction, including a remote control process $(\mathrm{n}=8375)$, was developed and introduced into the health care system of the Moscow Region. The ground for assessing the effectiveness of remote clinical management in 2019-2020 was the health care system of a big city. Based on the analysis of the protocols of endovascular procedures $(\mathrm{n}=2966)$, we studied the effectiveness of intraoperative decisions on treatment tactics after performing emergency coronary angiography (ECA) by interventional cardiologists. In 2019-2020 ( $\mathrm{n}=1875)$ established the assessment of the effectiveness of remote control based on a set of clinical and organizational parameters. The study of the effectiveness of intraoperative decision in the practice of interventional cardiologists was set on the analysis of the protocols of endovascular procedures $(n=2966)$. The subject of the assessment was the ratio of performed ECA to percutaneous coronary interventions (PCI).

The effectiveness of remote quality control of endovascular care was carried out on the foundation of the dynamics analysis of indicators for PCI in patients with acute myocardial infarction after coronary angiography.

The clinical effectiveness of remote quality control of endovascular care was assessed by the dynamics of acute myocardial infarction mortality. The research methods were statistical, mathematical and expert evaluation. The t-criteria was used to assess the reliability. Statistical processing of the material was carried out on the basis of the Statistica 6.0 package with the calculation of adequate statistical indicators and their reliability at $\mathrm{p}<0.05$.

\section{Results}

The results constituted a fragment of the study carried out in 2012-2020 on the foundation of the Moscow region health care systems [11]. Remote quality management of endovascular care included a complex of audiovisual communications and computer systemprocesses of operation, management, administration and the algorithm for making intraoperative decisions. Remote control was carried out via secure telecommunication channels by on-line video conferences, transmission of video clips and static images of angiograms.

The developed organizational and methodological foundations of remote quality control of endovascular care included some continuous medical education of interventional radiologists according to the developed educational programs (education), a secure telecommunication channel (communication), analysis and application of remotely obtained information in the operational process (integration), feedback and economic justification (effectiveness) and mentoring, including remote mentoring [12].

The organizational and methodological foundations of remote quality management of endovascular care, developed in 2012-2019 (figure 2).

Remote quality management of endovascular care was carried out on the basis of adherence to the developed algorithm of endovascular care (figure 3 ).

Professional education in the interventional radiology and continuing education are at the core of the quality and safety of endovascular care.

To improve the quality and safety of endovascular care according to the developed organizational and methodological algorithm, it is necessary to comply with the established documented criteria developed by the authors such as filling out the endovascular care protocol indicating its name, number, date and time;

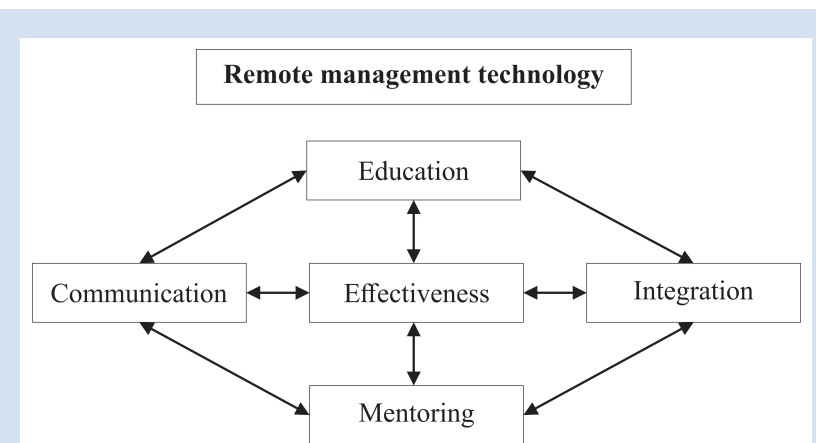

Figure 2. Organizational and methodological foundations of remote quality management of endovascular care

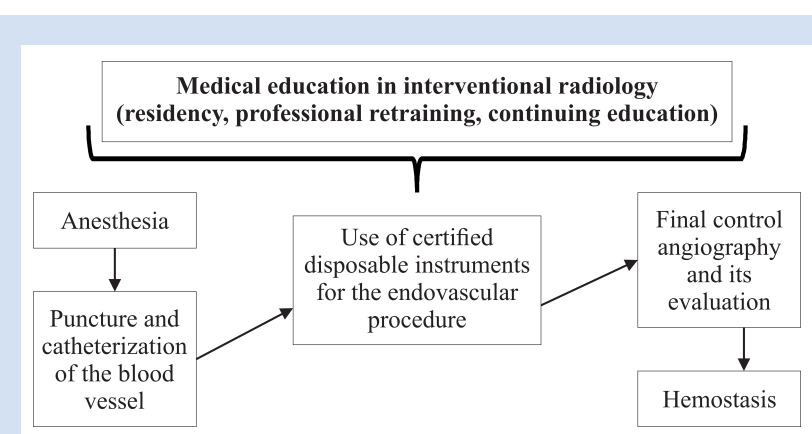

Figure 3. Algorithm of endovascular care 
the full name of the patient, the diagnosis and the department where the patient is being treated; the name of the operating surgeon, anesthesiologist, operating room nurse, anesthesiologist and anesthetist, a detailed and understandable description in the protocol of all actions of the interventional radiologist during the endovascular procedure, indicating the name of the disposable devices used, their physical characteristics (rigidity, presence of a hydrophilic coating), sizes, pressure and the time of balloon inflation during angioplasty and stent implantation into a blood vessel [12].

The number of diagnostic coronary angiography and $\mathrm{PCI}$ in patients with acute coronary syndrome performed in 2019, January-March 2020, and in April-December 2020 is shown in table 1.

The study found that for the period from April to December 2020 the frequency of PCI in patients with acute myocardial infarction in a medical organization increased by $17.7 \%$ compared to 2019 and by $27.2 \%$ compared to the period January-March 2020 - before the remote quality management of endovascular care technology was introduced. The ratio of the incidence of PCI and coronary angiography in acute myocardial infarction with an increase in the CT segment in 2020 was $98 \%$, in acute myocardial infarction without an increase in the CT segment was $85.8 \%$ and in unstable angina pectoris was $69 \%$. In $89 \%$ of invasive treatment of patients with acute myocardial infarction the strategy of endovascular treatment was discussed and accepted by the interventional radiologists and cardiologists who were at the workplace in the hospital and remotely by the head of the interventional radiology department and the head of the regional vascular center who acted as a mentor. The mentor of the "cardio team" was a qualified cardiovascular surgeon (an interventional radiologist with more than 15 years of clinical and organizational experience).

The treatment strategy for a patient with acute myocardial infarction was adopted on the analysis of the electrocardiogram, laboratory test results, hemodynamic parameters, coronary angiography results.

The task of the head of the vascular center was to enable the interventional radiologist to choose a treatment strategy and, if necessary, make reasonable adjustments remotely.
The effectiveness of choosing the correct strategy was $100 \%$. This contributed to an increase in the intensity of emergency endovascular care, an improvement in the quality of medical care for patients with acute myocardial infarction in a medical organization, and a decrease in hospital mortality from myocardial infarction from $9.7 \%$ in 2019 to $8.2 \%$ from April to December $2020(\mathrm{p}=0,005)$.

Integration of the remote clinical quality management has contributed to the full-time provision endovascular care.

\section{Discussion}

Historically skills and knowledge in medicine were acquired through targeted practice with patients. The debate on the existence of innate skills is speculation in light of the evidence that only hours spent in deliberate practice under the guidance of a mentor are the basis of the most advanced levels of knowledge and competence.

The process of training a doctor is lengthy and requires the presence of an experienced mentor who provides the learning process, forms feedback and attentiveness of the young doctor in relation to making mistakes. It takes thousands of hours of practice to reach the perfect level of knowledge.

We cannot compare our results to other studies due to the lack of open data of remote quality mentoring of endovascular care in acute coronary syndrome.

The remote control process should be based on the principles of empathy mentoring and should not disturb professional relationships. A prerequisite for effective remote control is feedback. Discussion both the preventive process of improving the quality and the complications based on the trust of team members and vertical administrative medical staff management.

The period of the COVID-19 pandemic is associated with increased stress on health care, including the provision of medical care for acute coronary syndrome. In a time of a shortage of interventional cardiologists and a period of increased professional burden on health care in connection with the pandemic of COVID-19 remote control must be based on telehealth technologies to strength the systemic effectiveness of endovascular care.

Number of coronary endovascular procedures and ratio of interventions to cases for patients with acute coronary syndrome performed in 2019, January-March 2020 and April-December 2020

\begin{tabular}{|c|c|c|c|}
\hline Endovascular procedure & 2019 & January-March 2020 & April-December 2020 \\
\hline Coronarography (n) & 885 & 349 & 641 \\
\hline PCI (n) & 477 & 155 & 459 \\
\hline PCI/Coronarography for ACS (\%) & $53.9 \%$ & $44.4 \%$ & $71.6^{*}$ \\
\hline 1) $\mathrm{PCI} /$ Coronarography for AMI+ST (\%) & $66 \%$ & $82 \%$ & $98 \% *$ \\
\hline 2) PCI/Coronarography for AMI-ST (\%) & $86 \%$ & $56 \%$ & $85.8 \%$ \\
\hline 3) $\mathrm{PCI} /$ Coronarography for Unstable angina (\%) & $44.2 \%$ & $28.8 \%$ & $69 \% *$ \\
\hline
\end{tabular}


The results of the research lie in the discovery of the effectiveness of clinical management of remote quality control of endovascular care and the scientific and methodological substantiation of the strategic role of the mentor.

\section{Conclusion}

The development of remote control and the algorithm of endovascular care to improve the effectiveness of clinical management quality of endovascular care by remote mentoring is the target of healthcare strength. In our research the integration of a system of professional telehealth communications in the continuum of applied technologies for patient care of those with acute myocardial infarction increased the frequency of PCI (1.33-fold) based on the results of ECA.
The evaluation of the effectiveness of remote quality control of endovascular care using telehealth technologies made it possible to single out the role of a mentor in making clinical and organizational decisions. The effectiveness of clinical management to help of cardio-teams to conduct invasive diagnostics of coronary arteries and assess the results, to choose a strategy and implement a treatment algorithm, and to use the most effective and safe tools and the tactics of performing an endovascular procedure in practice was increased.

\section{Conflict of interest}

The authors declare no conflict of interest.

\section{Acknowledgments}

The study had no sponsorship.

\section{Author Information Form}

Abramov Aleksey Yu., DMSi, Director of the Medical Institute of the Peoples' Friendship University of Russia, Moscow, Russian Federation; ORCID 0000-0001-7178-1260

Kicha Dmitry I., DMSi, Professor of the Department of Healthcare Organization, Drug Provision, Medical Technologies and Hygiene of the Faculty of Continuing Medical Education of the Medical Institute of the Peoples' Friendship University of Russia, Moscow, Russian Federation; ORCID 0000-0001-6529-372X

Goloshchapov-Aksenov Roman S., DMSi, Associate Professor at the Department of Cardiology, X-ray Endovascular and Hybrid Methods of Diagnosis and Treatment of the Faculty of Continuing Medical Education of the Medical Institute of the Peoples' Friendship University of Russia, Moscow, Russian Federation; ORCID 0000-0003-3085-7729

Rukodayny Oleg V., DMSi, MBA, Acting Head of the Department of Healthcare Organization, Drug Provision, Medical Technologies and Hygiene of the Faculty of Continuing Medical Education of the Medical Institute of the Peoples' Friendship University of Russia, Moscow, Russian Federation; ORCID 0000-0001-9134-7189

Nazarov Alexandr M., DMSi, Associate Professor, lecturer at the Department of Anesthesiology and Resuscitation of the Pediatric Faculty, Orenburg State Medical University, Orenburg, Russian Federation; ORCID 0000-0003-4975-9336

Koledinsky Anton G., Head of the Department of Cardiology, Endovascular and Hybrid Treatment of the Medical Institute of the Peoples' Friendship University of Russia, Moscow, Russian Federation; ORCID 0000-0001-7274-0276

\section{Author Contribution Statement}

$A A Y u$ - contribution to the concept and design of the study, editing, approval of the final version, fully responsible for the content $K D I$ - contribution to the concept and design of the study, manuscript writing, editing, approval of the final version, fully responsible for the content

$G$-ARS - contribution to the concept and design of the study, data collection and analysis, manuscript writing, approval of the final version, fully responsible for the content

$R O V$ - data interpretation, data collection and analysis, editing, approval of the final version, fully responsible for the content

$N A M$ - data interpretation, editing, approval of the final version, fully responsible for the content

$K A G$ - data interpretation, editing, approval of the final version, fully responsible for the content

\section{СПИСОК ЛИТЕРАТУРЫ}

1. Khan M.A., Hashim M.J., Mustafa H., Baniyas M.Y., Suwaidi S.K.B.M., AlKatheeri R., Alblooshi F.M.K., Almatrooshi M.E.A.H., Alzaabi M.E.H., Darmaki R.S., Lootah S.N.A.H. Global Epidemiology of Ischemic Heart Disease: Results from the Global Burden of Disease Study. Cureus. 2020;12(7):e9349. doi: 10.7759/cureus.9349.

2. Maingard J., Kok H.K., Ranatunga D., Brooks D.M., Chandra R.V., Lee M.J., Asadi H. The future of interventional and neurointerventional radiology: learning lessons from the past. Br J Radiol. 2017;90(1080):20170473. doi: 10.1259/ bjr.20170473.

3. Gillis N., Arslanian-Engoren C., Struble L. Acute Coronary Syndromes in Older Adults: A Review of Literature. Journal of Emergency Nursing. 2013; 40(3): pp. 270-275. doi: 10.1016/j.jen.2013.03.003.

4. EpiCast Report: Acute Coronary Syndrome ACS Epidemiology Forecast to 2025. Cardiovascular Week. 2014; p. 17. http://www.reportlinker.com/p01957646/EpiCast-Report- 
Acute-Coronary-Syndrome-ACS---Epidemiology-Forecastto-2022.html\#utm source=prnewswire\&utm medium=pr\&utm campaign $=$ Pathology

5. De Filippo O., D’Ascenzo F., Angelini F., Bocchino P.P., Conrotto F., Saglietto A., Secco G.G., Campo G., et al. Reduced rate of hospital admissions for ACS during Covid-19 outbreak in northern Italy. New Engl. J. Med. 2020; 383: pp. 88-89. doi: 10.1056/NEJMc2009166.

6. Garcia S., Albaghdadi M., Meraj P., Schmidt C., Garberich R., Jaffer F.A., Dixon S., Rade J.J., Tannenbaum M., Chambers J., Huang P.P., Henry T.D. Reduction in ST-segment elevation cardiac catheterization laboratory activations in the United States during COVID-19 pandemic. J. Am. Coll. Cardiol. 2020; 75(22): pp. 2871-2872. doi: 10.1016/j.jacc.2020.04.011.

7. Hall M., Bebb O., Dondo T., Yan A.T., Goodman S.G., Bueno H., Chew D.P., Brieger D., Batin P.D., Farkouh M.E., Hemingway H., Timmis A., Fox K.A.A., Gale C.P. Guidelineindicated treatments and diagnostics, GRACE risk score, and survival for non-ST elevation myocardial infarction. Eur Heart
J. 2018;39(42):3798-3806. doi: 10.1093/eurheartj/ehy517.

8. Odiorne G. Mentoring - an American management innovation. Personnel Administrator. 1985; 30:63-65.

9. Alred G., Garvey B., Smith R. The Mentoring pocketbook. United Kingdom: British Llibrary. Management pocketbook; $2010.128 \mathrm{p}$.

10. Demaerschalk B.M., Berg J., Chong B.W., Gross H., Nystrom K., Adeoye O., Schwamm L., Wechsler L., Whitchurch S. American Telemedicine Association: Telestroke Guidelines. Telemed J E Health. 2017;23(5):376-389. doi: 10.1089/ tmj.2017.0006.

11. Голощапов-Аксенов Р.С., Семенов В.Ю., Кича Д.И. Организационные и клинические основы рентгенохирургических методов диагностики и лечения сердечно-сосудистых заболеваний. М.: МИА; 2019. 365 с.

12. Goloshchapov-Aksenov R.S., Kicha D.I. The organizational and legal framework and history of X-ray endovascular care in the Russian Federation. Вопросы истории. 2020; 6: 179-184. doi: 10.31166/VoprosyIstorii202006Statyi13

\section{REFERENCES}

1. Khan M.A., Hashim M.J., Mustafa H., Baniyas M.Y., Suwaidi S.K.B.M., AlKatheeri R., Alblooshi F.M.K., Almatrooshi M.E.A.H., Alzaabi M.E.H., Darmaki R.S., Lootah S.N.A.H. Global Epidemiology of Ischemic Heart Disease: Results from the Global Burden of Disease Study. Cureus. 2020;12(7):e9349. doi: 10.7759/cureus.9349.

2. Maingard J., Kok H.K., Ranatunga D., Brooks D.M., Chandra R.V., Lee M.J., Asadi H. The future of interventional and neurointerventional radiology: learning lessons from the past. $\mathrm{Br}$ J Radiol. 2017;90(1080):20170473. doi: 10.1259/bjr.20170473.

3. Gillis N., Arslanian-Engoren C., Struble L. Acute Coronary Syndromes in Older Adults: A Review of Literature. Journal of Emergency Nursing. 2013; 40(3): pp. 270-275. doi: 10.1016/j.jen.2013.03.003.

4. EpiCast Report: Acute Coronary Syndrome ACS Epidemiology Forecast to 2025. Cardiovascular Week. 2014; p. $\quad 17 . \quad \mathrm{http}: / /$ www.reportlinker.com/p01957646/EpiCastReport-Acute-Coronary-Syndrome-ACS---EpidemiologyForecast-to-2022.html\#utm_source=prnewswire\&utm medium $=$ pr\&utm_campaign $=$ Pathology

5. De Filippo O., D’Ascenzo F., Angelini F., Bocchino P.P., Conrotto F., Saglietto A., Secco G.G., Campo G., et al. Reduced rate of hospital admissions for ACS during Covid-19 outbreak in northern Italy. New Engl. J. Med. 2020; 383: pp. 88-89. doi: 10.1056/NEJMc2009166.

6. Garcia S., Albaghdadi M., Meraj P., Schmidt C., Garberich R., Jaffer F.A., Dixon S., Rade J.J., Tannenbaum M., Chambers J., Huang P.P., Henry T.D. Reduction in ST-segment elevation cardiac catheterization laboratory activations in the United States during COVID-19 pandemic. J. Am. Coll. Cardiol. 2020; 75(22): pp. 2871-2872. doi: 10.1016/j.jacc.2020.04.011.

7. Hall M., Bebb O., Dondo T., Yan A.T., Goodman S.G., Bueno H., Chew D.P., Brieger D., Batin P.D., Farkouh M.E., Hemingway H., Timmis A., Fox K.A.A., Gale C.P. Guidelineindicated treatments and diagnostics, GRACE risk score, and survival for non-ST elevation myocardial infarction. Eur Heart J. 2018;39(42):3798-3806. doi: 10.1093/eurheartj/ehy517.

8. Odiorne G. Mentoring - an American management innovation. Personnel Administrator. 1985; 30:63-65.

9. Alred G., Garvey B., Smith R. The Mentoring pocketbook. United Kingdom: British Llibrary. Management pocketbook; 2010.128 p.

10. Demaerschalk B.M., Berg J., Chong B.W., Gross H., Nystrom K., Adeoye O., Schwamm L., Wechsler L., Whitchurch S. American Telemedicine Association: Telestroke Guidelines. Telemed J E Health. 2017;23(5):376-389. doi: 10.1089/ tmj.2017.0006.

11. Goloshchapov-Aksenov R., Semenov V., Kicha D. Organizational and clinical bases of endovascular treatment of cardiovascular diseases. Moscow: Medical informational agency; 2019. 365 p. (In Russian)

12. Goloshchapov-Aksenov R.S., Kicha D.I. The organizational and legal framework and history of X-ray endovascular care in the Russian Federation. Voprosy istorii. 2020; 6: 179-184. doi: 10.31166/VoprosyIstorii202006Statyi13 (In Russian)

To cite: Abramov A.Yu., Kicha D.I., Goloshchapov-Aksenov R.S., Rukodayniy O.V., Nazarov A.M., Koledinsky A.G. Remote clinical quality management of endovascular care. Complex Issues of Cardiovascular Diseases. 2021;10(4): 106-111. DOI: 10.17802/2306-1278-2021-10-4-106-111 